\title{
LOS MUNDOS VIRTUALES DE APRENDIZAJE COMO MÉTODO DE ENSEÑANZA EN LA UNIVERSIDAD TÉCNICA DE BABAHOYO
}

\section{VIRTUAL WORLDS AS LEARNING IN TEACHING METHOD IN THE TECHNICAL UNIVERSITY OF BABAHOYO}

Raúl Armando Ramos Morocho ${ }^{1}$

1. Universidad Técnica de Babahoyo. Facultad de Administración, Finanzas e Informática. Babahoyo, Los Ríos (Ecuador). E-mail: rramos@utb.edu.ec

Citación sugerida:

Ramos Morocho, R.A. (2016). Los mundos virtuales de aprendizaje como método de enseñanza en la Universidad Técnica de Babahoyo. 3C TIC: Cuadernos de desarrollo aplicados a las TIC, 5(4), 63-87. DOI: <http://dx.doi.org/10.17993/3ctic.2016.54.63-87/>. 


\section{RESUMEN}

Los mundos virtuales se han convertido en algo cotidiano, su uso va desde el entretenimiento hasta la simulación de entornos científicos reales; la masificación del uso de computadores en casi todas las actividades humanas ha permitido que esta tecnología ingrese a los hogares comunes y por ende también a las instituciones educativas de todos los niveles.

En ese contexto, en el presente artículo se describe la relación existente entre la utilización de mundos virtuales de aprendizaje y los métodos tradicionales de enseñanza; para diferenciar el nivel de captación de los contenidos impartidos por los docentes al utilizar distintas metodologías, se creó un curso virtual (Mundo Virtual) para la asignatura redes de computadores en la escuela de sistemas de la Universidad Técnica de Babahoyo para lo que se utilizó una combinación de plataformas MOODLE, SLOODLE y SECOND LIFE, tomando para el estudio como población los treinta y seis estudiantes del paralelo " $A$ " y los treinta y cuatro alumnos del paralelo " $B$ " que para este caso es el grupo de control que utilizó solamente los métodos formales tradicionales de educación.

Antes de poner en ejecución la propuesta, se determinó que los dos grupos estén en condiciones similares. Para ello se tomaron los resultados de la evaluación final de semestre, es decir los promedios finales de cada alumno en la Asignatura de Redes de Computadores y luego de aplicar z normalizado se pudo determinar que los estudiantes que utilizan Plataformas Virtuales en el proceso de aprendizaje de la asignatura Redes de Computadores supera el nivel de rendimiento Académico de los estudiantes que no las utilizan.

\section{ABSTRACT}

Virtual worlds have become commonplace, its use goes from entertainment to real scientific simulation environments; the mass use of computers in almost all human activities has allowed this technology to enter ordinary households and therefore also to educational institutions of all levels.

In this context, in this article the relationship between the use of virtual worlds for learning and traditional teaching methods described; to distinguish the level of uptake of the material taught by teachers using different methodologies contents, a virtual course (Virtual World) for the subject computer networks in the school systems of the Technical University of Babahoyo for what was created was used a combination of platform MOODLE, SLOODLE and SECOND LIFE, taking to the study as a population of thirty-six students from parallel "a" and thirty-four students from parallel "B" which in this case is the control group that used only traditional formal methods of education.

Before implement the proposal, it was determined that the two groups are in similar conditions. To do this the results of the final evaluation of semester were taken, ie the final 
average of each student in the Course of Computer Networks and after applying standardized $\mathrm{z}$ was determined that students using Virtual Platforms in the learning process Computer Networks subject exceeds the level of academic achievement of students who do not use them.

\section{PALABRAS CLAVE}

Mundos Virtuales, SLOODLE, MOODLE, Second Life, Educación Virtual.

\section{KEY WORDS}

Virtual Worlds, SLOODLE, MOODLE, Second Life, Virtual Education. 


\section{INTRODUCCIÓN}

Cualquier proceso de investigación, se inicia en torno a un problema al que no hemos dado una solución o respuesta adecuada. Un tema de profundo interés para un profesor Universitario es saber cómo adecuar los contenidos del currículo a las nuevas tecnologías, así como también saber cuál es la metodología más adecuada para cada actividad, para cada materia y para cada nivel del proceso de enseñanza-aprendizaje.

La cumbre Mundial sobre la Sociedad de la Información (CMSI), reunida en Túnez en noviembre de 2005, en su declaración de principios, manifestó que "las Tecnologías dela Información y la Comunicación (TIC) poseen enormes posibilidades para acrecentar el acceso a una educación de calidad, así como para facilitar el proceso mismo de aprendizaje, sentando de esta forma las bases para la creación de una Sociedad de la Información abierta a todos y orientada al desarrollo y el conocimiento"

En este contexto, podemos plantearnos la utilización de la Educación Virtual como recurso educativo: los mundos virtuales de aprendizaje podrían convertirse en una alternativa viable y rápida que pudiera dar respuesta a los nuevos retos que se nos plantean, permitiéndo la apertura de nuevos escenarios de colaboración, distintos a los espacios físicos formativos tradicionales.

Utilizar nuevos contenidos y formatos educativos y aprovechar la flexibilidad que nos aporta la asincronía temporal y la deslocalización física del grupo, encontrando aquellos momentos que mejor se adapten a las necesidades y accediendo a los recursos desde diferentes ubicaciones.

Los mundos virtuales de aprendizaje surgen con la finalidad de extender, mejorar y hacer más eficiente la forma de enseñanza y potenciar de manera eficaz el aprendizaje a través de escenarios virtuales inmersivos, como elemento innovador donde se incluyen recursos de aprendizaje enriquecidos por teorías y enfoques pedagógicos actuales, capacidades para el trabajo colaborativo y cooperativo.

\section{REVISIÓN BIBLIOGRÁFICA}

\subsection{MUNDOS VIRTUALES}

En la actualidad la tecnología está permitiendo el desarrollo de espacios digitales que simulan el entorno físico de la realidad, y que pueden ser utilizados para llevar a cabo algunas actividades humanas, entre ellas la educación. Este tema es muy actual, centrando la atención de distintos campos científicos, tal como puede comprobarse en distintas publicaciones recientes (Good, Howland y Thackray, 2008; Castaño Garrido, 2008; Jamaladin, Chee y Ho, 2015; Girvan y Savage, 2010; entre otros). 
¿Qué son los mundos virtuales? Para responder a esta cuestión se han tomado en consideración múltiples autores que definen de manera distinta pero con características comunes a estos entornos de inmersión, una de las definiciones que más llamó la atención por su carácter abarcador y facilidad de comprensión es:

"Un mundo virtual es un género de comunidad en línea que a menudo toma la forma de un entorno simulado por ordenador, a través de la cual los usuarios pueden interactuar entre sí, así como utilizar y crear objetos. Los mundos virtuales están destinados a que sus usuarios los habiten e interactúen, y el término se ha convertido hoy en gran parte sinónimo de entornos interactivos 3D virtual, donde los usuarios adoptan la forma de avatares gráficamente visible para los demás. Estos avatares son generalmente descritos como texto, bidimensional, tridimensional o representaciones gráficas, aunque son posibles otras formas (auditiva y sensaciones táctiles, por ejemplo). Algunos, pero no todos, los mundos virtuales permiten usuarios múltiples..." (Caws \& Hamel, 2016, p. 19).

Según esto un Mundo Virtual es un espacio digital que simula una realidad. Los Mundos Virtuales se conocen como Ambientes Multiusuario Virtuales (AMU's) o Multiuser Virtual Environments (MUVE's) en inglés. Los AMU's son "no solamente un sistema que permite la participación, sino que se trata de un espacio colaborativo de alto nivel, de simulación, de comprobación de hipótesis, de interacción, de creatividad y despeño (Antonio Matas Terrón \& Miguekl Angel Ballesteros Moscosio, 2011).

El desarrollo de los MV ha evolucionado gracias a la tecnología que ha permitido también el desarrollo de los juegos de simulación, principalmente los llamados Juegos Masivos Multijugador En-línea (Massive Multiplayer Online Games) tales como Dragones y Mazmorras(http://www.dragonesymazmorras.net) o World of Worcraft (http://www.wowesp.com). No obstante los MV no deben confundirse con un juego en-línea multiusuario, aunque algunos juegos se desarrollen en MV.

La empresa Linden World creó en 2002 el Mundo Virtual Second Life. La aparición de Second Life (SL) demostró el interés social de este tipo de herramientas así como su potencial para atraer a empresas e instituciones.

El desarrollo de ambientes educativos en los MV's se produjo pronto. El mismo SL creó una red para adolescentes (Teen Grid), abierta solamente para jóvenes de 13 a 17 años. Actualmente, SL cuenta con más de 200 instituciones de enseñanza superior y 3000 miembros participando en su lista Second Life Educators.

Entre las instituciones que utilizan los mundos virtuales de aprendizaje se pueden mencionar entre otras:

La Universidad de California que ha creado un hospital para enseñar cómo viven sus pacientes la esquizofrenia.

La UNIR creo un campus virtual completo para sus estudiantes de modalidad a distancia pero que también es utilizado por los alumnos presenciales. 
La universidad Carlos III de Madrid también tiene implementado un mundo virtual para la participación interactiva de sus estudiantes.

Características de los mundos virtuales como recursos educativos

En este tipo de ambientes aparecen tres elementos que son básicos para cualquier comunidad de aprendizaje, que tal como afirman Garrison, Anderson y Archer (2000) son los siguientes:

- Presencia social: La presencial social en enseñanza en-línea ha sido descrita como la habilidad del alumnado de proyectarse social y emocionalmente. Los alumnos son percibidos por el grupo como "personas reales" a través de una comunicación mediada por la tecnología. En enseñanza virtual existen serias dificultades para conseguir esta proyección, no solamente social, sino principalmente emocional, tanto del alumnado como del profesorado. Sin embargo, las posibilidades que ofrecen los avatares en interfaces 3D puede facilitar esta proyección social y emocional.

- Presencia cognitiva: Consiste en el proceso por el cual los participantes pueden construir y confirmar su conocimiento. Son esenciales las actividades que permiten analizar la información, sintetizarla, reflexionar individualmente sobre ella así como compartir estas reflexiones a través del debate crítico.

- La interacción: La interacción es un elemento básico de la enseñanza virtual, sin embargo no es suficiente para que se produzca un aprendizaje efectivo. En este sentido, los docentes son facilitadores del aprendizaje que orientan el proceso. En un ambiente virtual son especialmente útiles las interacciones a través de debates y discusiones sobre el contenido que se está trabajando.

Los sistemas 3D VLE actúan como entornos globales de aprendizaje donde el usuario (el alumnado y el profesorado) deben interaccionar según las reglas marcadas (programadas) en ese MV. Estas condiciones de interacción están estrechamente ligadas al concepto "affordance". Originalmente Gibson (1979, cit., Girva, y Savage, 2010) describió affordance como la relación que se establece entre un organismo y aquello que ofrece un ambiente. Se trata de una idea propiamente del ámbito de la biología y la ecología, que ha sido reinterpretada en el ámbito de la tecnología educativa. Así, Kirschner (2002) define específicamente "Educational Affordance" de una tecnología como "la característica de un artefacto que determina si, y cómo un particular comportamiento aprendido podría ser puesto en marcha dentro de un contexto dado" ${ }^{26}$. Esta cuestión tiene bastante relación con algunas teorías de la Psicología Cognitiva. Principalmente con la teoría de esquemas y con la teoría de escenarios ${ }^{27 .}$

¿Cuándo se puede considerar que un MV es educativo? Un elemento básico de la acción educativa es el desarrollo íntegro de la persona, lo que incluye el desarrollo de función crítica, es decir, la capacidad de valorar de forma reflexiva y constructiva tanto la acción de los demás como la propia con relación a la sociedad.

\footnotetext{
${ }^{26}$ Kirschner, 2002: 19

${ }^{27}$ Rodríguez, 1987
} 
En este sentido, un sistema es educativo cuando se utiliza para desarrollar una actividad planificada, intencionada y estructurada, que pretende lograr el desarrollo integral del individuo. La educación implica aprendizaje, pero el aprendizaje estricto (adquisición de conocimientos) no siempre implica educación.

Los componentes educativos de un MV son:

- No sólo se reduce al aprendizaje de conceptos o procedimientos.

- Se han planificado las actividades, tiempos y recursos.

- Se han estructurado los contenidos, conceptos, procedimientos, actitudes y competencias que se trabajan en las actividades.

- Y existe la intención de conseguir el desarrollo integral de la persona, incluyendo su capacidad de crítica constructiva hacía sí mismo y hacia las acciones de los demás.

Todos estos componentes tienen sentido y adquieren eficacia cuando son estructurados y articulados dentro de una acción coherente que se sustenta sobre un modelo formativo.

En este sentido el constructivismo parece ser un modelo que se ajusta bastante bien a lo que sucede en el aprendizaje en entornos virtuales ${ }^{28}$. Sin embargo, este aprendizaje se realiza dentro de un entorno social, inmerso en una cultura. La persona que aprende está sometida a una interacción con su medio social, de forma que el conocimiento que elabora es resultado de no solamente del proceso de acomodación y asimilación, sino también de como el hablar con los demás, debatir, escuchar las opiniones de otros, asumir las críticas o aportarlas hacia los comentarios de los demás, van matizando, depurando, y dando forma definitiva a ese conocimiento que se integra en el esquema cognitivo de cada individuo. Este constructivismo que tienen en cuenta la acción cultural, la acción del entorno, se suele conocer como "constructivismo social". Existe otro modelo que ha evolucionado a partir de las propuestas del constructivismo, se trata del "constructivismo comunal"29. Este modelo es un enfoque de aprendizaje que hace hincapié no solamente en la construcción del conocimiento por parte de cada individuo (constructivismo), dentro de un entorno cultural y por tanto mediado por el mismo (constructivismo social) sino también en el hecho de que cada persona elabora conceptos, genera información, aporta procedimientos, difunde actitudes, etc., y todas estas "elaboraciones", "artefactos", u "objetos de conocimiento", es decir, todo estos contenidos conceptuales los vuelca a la sociedad para uso y beneficio de sus miembros. Estudios recientes han tratado este tema, partiendo y comprobando la plausibilidad de la hipótesis de que "las características de la Construcción del Conocimiento y del Constructivismo Comunal promueven oportunidades para aprovechar la gama de affordances que ofrece Second Life" 30 .

\footnotetext{
28Seitzinger, 2006; Hargis, 2008

${ }^{29}$ Holmes, Tangney, FitzGibbon, Savage y Mehan, 2001

${ }^{30}$ Girvan y Savage, 2010: 344
} 


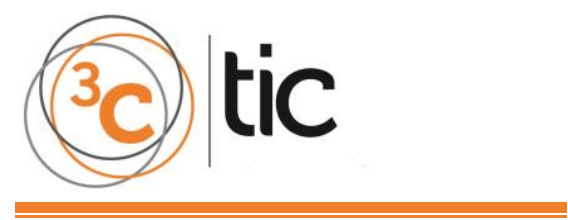

No obstante, estos estudios llegan a la conclusión de que las posibilidades previstas por el constructivismo comunal depende de la naturaleza y flexibilidad de los MV donde se desarrollen.

\subsection{EL APRENDIZAJE EN LOS MV}

Sin embargo, aunque el aprendizaje se realice en entornos virtuales, en espacios simulados, o debajo de una choza, dentro de la persona siguen dándose los mismos mecanismos neuropsicológicos que hace miles de años. En definitiva, el proceso de aprendizaje de la persona es el mismo de siempre aunque el medio donde se desarrollo esté cambiando continuamente.

En los MV, compartimos la idea de Carlos Marcelo (2009) en una entrevista a Learning Review, que ante la pregunta de la entrevistadora "¿Qué rol debe asumir el formador dentro de una entorno 3D?" respondía:

"La experiencia que hemos acumulado a lo largo de este año, nos permite asegurar que el rol el formador en un entorno 3D cada vez se puede parecer más a las diferentes posibilidades metodológicas y de comunicación que tenemos en la formación virtual’”31

Por tanto, el primer elemento a tener en cuenta en el diseño de una acción educativa en un MV es identificar qué enseñar. De forma clásica, el qué enseñar se suele dividir en conceptos, procedimientos y actitudes:

Conceptos: En un MV la enseñanza de conceptos, definiciones, datos, fechas, etc., se puede realizar a través de anotaciones, material escrito, presentaciones multimedia, etc., donde se ofrece al alumnado todo el cuerpo de información que necesitará comprender, asimilar, y memorizar (su significado), para poder afrontar eficazmente su formación. Los MV tienen implementados suficientes recursos para satisfacer la exposición de texto, vídeos, presentaciones, gráficos, etc.

Procedimientos: La enseñanza de los procedimientos son los grandes beneficiados al utilizar un entorno 3D. En la enseñanza presencial real de algunas materias (como química, física, ingeniería, educación, etc.) no es posible llevar a cabo algunos procedimientos porque los resultados pueden ser nefastos para la integridad física o psicológica de los participantes. Por ejemplo, no es razonable que un químico experimente en clase con ácido nítrico, ácido sulfúrico y glicerina porque los resultados pueden ser fatales (se trata de los compuestos de la nitroglicerina). Tampoco es razonable que un estudiante de psicología trate de aplicar un programa de extinción de conducta a un menor, en la realidad, solamente para que el

${ }^{31}$ Carlos Marcelo (2009) 
estudiante practique el procedimiento. En este sentido los 3D VLE integran sistemas de simulación, que permiten representar con cierto grado de realismo (cada vez más) situaciones donde practicar los distintos procedimientos, sin sufrir sus peores consecuencias. En este caso el recurso básico es la simulación. La construcción de simulaciones efectivas tiene que ver con la construcción de representaciones de la realidad que integren los elementos básicos del sistema real que se trata de representar ${ }^{32}$.

Actitudes: En esta dimensión debe incluirse también los aspectos emocionales y afectivos. De hecho las actitudes deben ser consideradas como un elemento complejo, no se trata de transmitir solamente una predisposición hacia un tema, sino potenciar un estado de ánimo positivo cuando la persona esté realizando una tarea formativa ${ }^{33}$. En este sentido los MV también suponen, y lo será aún más en el futuro, toda una revolución en la formación actitudinal a través de la teleformación. La transmisión de las actitudes, de los valores, de las emociones, las consigue el docente principalmente, con su lenguaje no verbal, es decir, con todas las inflexiones de la voz donde pone énfasis a lo más importante de su mensaje, con su mirada cuando se fija en unos aspectos un otros de lo que explica, cuando mira a un alumno u otro dándoles el turno de palabra, cuando eleva su volumen al hablar de algo, etc. El docente transmite actitudes cuando es capaz de transmitir la emoción que le provoca su trabajo.

Hasta el momento una de las grandes deficiencias de los entornos de teleformación es su incapacidad de transmitir eficazmente estas emociones. Sin embargo, la utilización de avatares en 3D, con capacidad expresiva, gracias al software que los genera, facilita la expresión de algunas emociones básicas. En el futuro será posible que estas criaturas digitales transmitan con sus gestos y movimientos, un amplio conjunto de indicadores emocionales, y con ello contribuir al desarrollo de actitudes en el alumnado.

Además de qué enseñar, también debe atenderse al grado de competencia que se pretende conseguir en el alumnado con relación a los contenidos:

- Nivel tácito: Es en nivel de competencia más básico, donde se exige menos recursos por parte del alumnado. Básicamente consiste en pedir al discente que repita o exponga aquellos contenidos que ha aprendido previamente. Para ello solamente tienen que recordar dichos contenidos, principalmente conceptos, definiciones y datos, expresándolos en sus propias palabras, según lo que ha entendido de dicho contenido. Es decir, que exponga el resultado de la acomodación-asimilación que ha experimentado. El proceso de aprendizaje es relativamente simple, consiste en exponer a los objetos del MV donde el alumnado pueda leer, ver y oír dichos contenidos. El papel delos docentes

\footnotetext{
${ }^{32}$ Matas, 2008

${ }^{33}$ Matas, 2003
} 
consiste en elegir dichos contenidos e incluirlos en el MV en distintos formatos (visual, auditivo y textual).

- Nivel de aplicación: De nuevo aquí los MV demuestran su potencial. En este nivel del alumnado demuestra la capacidad de resolver problemas a partir del conocimiento y recursos que tiene.

- El potencial de los entornos 3D de enseñanza virtual para simular situaciones problemáticas, facilita el aprendizaje del alumnado a través de la resolución de casos. El docente en este caso debe proponer casos que sean pertinentes al contenido y que tengan sentido para el alumnado.

Nivel de creación: Un nivel superior de competencia es aquel donde el alumnado es capaz de elaborar su propio contenido. Aquí adquiere sentido lo que se exponen anteriormente con relación al constructivismo comunal. La creación de contenidos se puede realizar a partir de actividades programadas por los docentes que requieran de la aportación del alumnado para su resolución. Los MV tiene recursos suficientes para que este conocimiento elaborado por el alumnado sea compartido por el grupo ${ }^{34}$.

- Nivel de evaluación: Se trata de un nivel de competencia de experto. El alumnado tendrá que valorar críticamente tanto su trabajo como el de los demás. Las comunidades de aprendizaje requieren de este nivel de competencia, y los MV aportan un entorno controlado ideal para desarrollarlo. Un entorno virtual 3D donde los participantes pueden "verse" y "hablarse" entre sí, facilitan los debates y las discusiones académicas sincrónicas.

De esta forma los MV superan a las plataformas de teleformación permitiendo una interacción basada en la presencia virtual, con sistemas de comunicación eficientes que van más allá del "chat". Como se decía anteriormente, las ventajas de la interacción a partir de avatares mejorará al mismo tiempo que avance la sensación de realidad que se consiguen en los entornos virtuales.

\subsection{FORMACIÓN A DISTANCIA}

La formación a distancia tiene su origen a finales del siglo XIX en Gran Bretaña, en el ámbito universitario, destinado a los trabajadores profesionalmente cualificados. Los orígenes de la historia moderna de la educación a distancia se remontan a 1840, año en el que Sir Issac Pitman comenzó a utilizar el correo para impartir cursos de estenografía por correspondencia en Gran Bretaña. Según afirma Matthews, la fundación de la Open University británica en 1971 marca el inicio de una segunda fase en el desarrollo de la educación a distancia, en la

\footnotetext{
${ }^{34}$ Girvan y Savage, 2010
} 


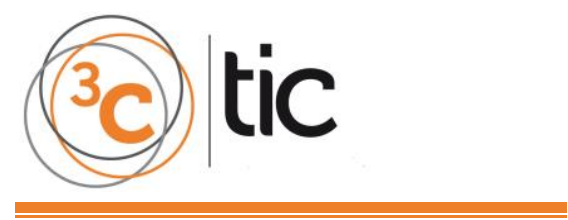

cual se utilizan diversos medios tanto para la comunicación con los estudiantes como para la recepción y envío de los materiales educativos.

Más tarde la iniciativa fue exportada a otros países en proceso de modernización. Hoy en día, en los Estados Unidos, la mayor parte de las más de cuatro mil instituciones de enseñanza superior acreditadas por el Departamento de Estado ofrecen algún tipo de cursos a distancia. Actualmente, existen más de 1500 instituciones en todo el mundo de educación a distancia que superan ya los 30 millones de estudiantes.

Con una compleja y específica metodología y adaptación curricular, la formación a distancia se está consolidando como un sistema abierto y flexible que incorpora innovadores sistemas de evaluación. Por ello, cada día es más usual y necesaria la utilización de producciones audiovisuales, materiales multimedia, tecnologías de la información y la comunicación y su aplicación en el proceso de enseñanza- aprendizaje.

La historia del desarrollo de materiales didácticos para la Web ha discurrido de forma paralela a la evolución de este medio. La rápida expansión de internet ocurrida en todos los niveles de la sociedad también se ha reflejado en el ámbito educativo puesto que la explotación didáctica de la Web permite ampliar la oferta educativa, la calidad de la enseñanza y el acceso a la educación. Sin embargo, el desarrollo de materiales didácticos para la Web no puede ser exclusiva responsabilidad de esfuerzos individuales.

Para hacer frente a esta necesidad, han comenzado a aparecer en el mercado desde mediados de los años noventa plataformas integradas para la creación de cursos completos para la Web. Las diferencias principales que existen entre ellas radican en el precio de las licencias de uso, en el abanico de recursos que ofrecen tanto al diseñador/gestor de los cursos como a los estudiantes y en los requerimientos tecnológicos para su instalación y mantenimiento.

\subsection{MODALIDADES DE FORMACIÓN A DISTANCIA}

Según la metodología empleada, en función de los medios, las herramientas de trabajo o los materiales utilizados, podemos distinguir diferentes modalidades. Las metodologías utilizadas en cada una de las modalidades nombradas, a menudo son complementarias, es decir que la utilización de una no excluye la compatibilidad con otra.

\section{- Formación a distancia no presencial}

El proceso más usual, una vez el alumno ya se ha inscrito al curso que desea, es que se le asigne un tutor, se le envíen los materiales del curso y se ponga a trabajar en sus contenidos.

Cada cierto tiempo, deberá enviar las actividades y los ejercicios que haya realizado, para que el tutor los corrija y le haga los comentarios que considere oportunos. El tutor guía su proceso de aprendizaje, y se pone en contacto con el alumno vía teléfono, carta, fax o correo electrónico (e-mail). El alumnado interactúa con otros alumnos y con el profesor, 
normalmente, mediante correo electrónico o chat realizando los trabajos y exámenes que se le proponen. Actualmente, el material didáctico y los recursos utilizados acostumbran a facilitarse on-line.

- El E-learning (de Electronic Learning) consiste en una enseñanza a distancia en la que predomina una comunicación de doble vía asíncrona donde se usa preferentemente Internet como medio de comunicación y de distribución del conocimiento, de tal manera que el alumno es el centro de una formación independiente y flexible, al tener que gestionar su propio aprendizaje, generalmente con ayuda de tutores externos. Una solución e-learning está conformada por tres elementos fundamentales: Plataforma, Contenidos y Herramientas comunicativas.

Desde mediados de los años noventa han empezado a desarrollarse plataformas integradas para el desarrollo de materiales en la web, que permiten diseñar cursos virtuales de enseñanza asistida por ordenador, más conocidas como:

Plataformas virtuales, entornos de aprendizaje integrados o LMS (Learning Management Systems)

\section{- Formación a distancia semi-presencial}

Consiste en destinar parte del tiempo de estudio a asistir, tanto en grupo como individualmente, a tutorías de soporte con profesorado especializado, o a reuniones con otros estudiantes. El objetivo de estas reuniones es el de trabajar conjuntamente o el de aclarar dudas.

\section{- El B-Learning (formación combinada, del inglés blended learning)}

Consiste en un proceso docente semipresencial; esto significa que un curso en este formato incluirá tanto clases presenciales como actividades de e-learning.

Este modelo de formación hace uso de las ventajas de la formación $100 \%$ on-line y la formación presencial, combinándolas en un solo tipo de formación que agiliza la labor tanto del formador como del alumno. El diseño instruccional del programa académico para el que se ha decidido adoptar una modalidad b-Learning deberá incluir tanto actividades on-line como presenciales, pedagógicamente estructuradas, de modo que se facilite lograr el aprendizaje buscado.

\section{- Webs Docentes}

Un web docente no es un sitio en el que queremos mostrar una mera exposición de contenidos sobre un tema de nuestro interés, ni pretendemos únicamente informar a los visitantes sobre un listado de recursos para realizar una actividad.

Es un sitio web que ayude a los alumnos a alcanzar unos objetivos pedagógicos, para que al terminar su visita hayan incorporado determinados conceptos, manejen con soltura ciertos procedimientos $y$ hayan adquirido o afianzado ciertas actitudes. 
- Los contenidos en formato web permiten a los alumnos que sean ellos quienes estructuren su aprendizaje, seleccionando qué examinarán antes, después y de qué prescindirán.

- Facilita la interacción entre profesor-alumno y entre los propios alumnos.

- La inclusión de enlaces en la web permiten disponer de una cantidad impresionante de información al alcance de un clic.

- Los contenidos se actualizan fácilmente y con un coste inferior al de los materiales impresos.

- Facilita el acceso a los contenidos de la asignatura en horarios diferentes a la clase habitual. Puede incrementar la motivación al estudio de la asignatura.

- Es posible la inclusión de elementos multimedia (vídeos, imágenes, sonidos, animaciones, ...) que faciliten el estudio de la asignatura.

- El profesor podrá ir actualizando cada año la programación de la asignatura en función de las características de cada curso.

- La web docente va a permitir la creación progresiva de una base de datos de recursos, con apuntes, bibliografía y webs de interés para cada uno de los temas de la asignatura, lo cual facilita enormemente el aprendizaje del alumno.

- El mantenimiento de un sitio web es muy costoso en tiempo, ya que si queremos actualizar los contenidos con regularidad, comunicarnos con alumnos y profesores y seguir mejorando el diseño, el tiempo empleado es muy elevado.

- Se requiere que todos los alumnos tengan conocimientos previos de Internet y correo electrónico para poder aprovechar las ventajas de la web.

- La necesidad de formación del profesorado en el área de diseño de páginas web.

- Si se trata de educación a distancia, se requiere que los alumnos tengan conexión a Internet en sus casas para facilitar el acceso. Cada vez hay más usuarios de Internet, aunque no todos los alumnos tienen la conexión en sus casas.

\section{METODOLOGÍA}

\subsection{MÉTODOS, TÉCNICAS E INSTRUMENTOS DE RECOLECCIÓN DE DATOS}

Para la realización de la prueba se creó un mundo virtual de aprendizaje. Se utilizó SECOND LIFE y SLOODLE para interaccionar con la plataforma institucional MOODLE. Hay que destacar que el curso de redes de computadores ya estaba creado por lo que solamente hizo falta incrustar las extensiones necesarias para la integración de MOODLE con los metaversos de SLOODLE Y SECOND LIFE.

En SECOND LIFE se creó con la ayuda del SANDBOX un sitio de prueba. Debido a que no se realizó ningún pago existieron algunos inconvenientes ya que una vez pasado el período de prueba los metaversos perdían su configuración. Se compraron algunos componentes como una Aula y proyectores virtuales pero también se desarrollaron algunos componentes como bancas y escritorios. 


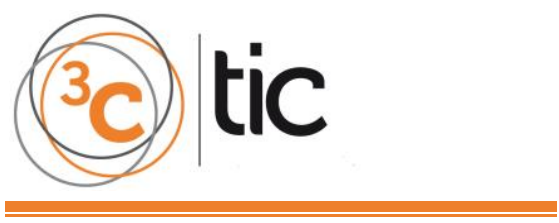

Para la recolección de la información se utilizaron instrumentos de evaluación de rendimiento académico tales como, foros, chats, test, laboratorios, talleres y wikies a los estudiantes de los dos paralelos según corresponda al modo virtual o presencial.

Para la prueba de hipótesis se utilizó la técnica estadística z normalizada.

\subsection{DISEÑO Y TIPO DE ESTUDIO}

Es un estudio cuasi - experimental con un grupo de control.

\subsection{DETERMINACIÓN DE LA POBLACIÓN Y MUESTRA}

La población está conformada por todos los estudiantes de la Escuela de Sistemas y Tecnologías de la Universidad Técnica de Babahoyo. Se toma en consideración los estudiantes del quinto semestre; 36 estudiantes del paralelo " $A$ " y los 34 alumnos del paralelo "B" que para este caso es el grupo de control, este grupo de control utilizó únicamente los métodos tradicionales de aprendizaje.

\subsection{PLANTEAMIENTO DE LA HIPÓTESIS}

3.4.1. Hipótesis General:

La utilización de mundos virtuales de aprendizaje permitirá mejorar el nivel de rendimiento académico de la Asignatura de Redes de Computadores en la Escuela de Sistemas y Tecnologías de la Universidad Técnica de Babahoyo.

\subsection{Determinación de las variables}

- Utilización de mundos virtuales de aprendizaje.

- Nivel de rendimiento académico.

\section{RESULTADOS}

Los datos que a continuación se muestran se obtuvieron aplicando la evaluación durante el semestre tanto al paralelo A como al paralelo B. Estos instrumentos se aplicaron bajo condiciones similares.

En cada uno de los instrumentos de evaluación se tomó en consideración los indicadores que se pretendían medir, cada una de las herramientas tiene una valoración sobre 10 puntos. 


\subsection{RESULTADOS OBTENIDOS DE LA APLICACIÓN DEL INSTRUMENTO DE EVALUACIÓN}

\subsubsection{Datos obtenidos del Paralelo A}

Los datos que se muestran a continuación se obtuvieron aplicando los instrumentos de evaluación pertinente a los 36 alumnos del paralelo " $A$ ".

Tabla 1. Datos Obtenidos con el paralelo A, al aplicar la evaluación semestral.

\begin{tabular}{|c|c|c|c|c|c|c|c|c|c|c|c|c|c|c|c|c|c|c|}
\hline № de Estudiante & 1 & 2 & 3 & 4 & 5 & 6 & 7 & 8 & 9 & 10 & 11 & 12 & 13 & 14 & 15 & 16 & 17 & 18 \\
\hline \multicolumn{19}{|l|}{ INSTRUMENTO/INDICADOR } \\
\hline $\begin{array}{l}\text { Laboratorios (ANALISIS Y } \\
\text { APLICACION) }\end{array}$ & 9,0 & 10,0 & 8,0 & 9,0 & 7,0 & 9,0 & 10,0 & 9,0 & 8,0 & 7,0 & 9,0 & 10,0 & 9,0 & 8,0 & 9,0 & 9,0 & 10,0 & 9,0 \\
\hline Foros (RAZONAMIENTO) & 10,0 & 10,0 & 9,5 & 8,0 & 9,0 & 9,5 & 9,0 & 9,0 & 9,5 & 10,0 & 9,0 & 9,0 & 10,0 & 9,0 & 9,0 & 9,0 & 10,0 & 9,5 \\
\hline $\begin{array}{c}\text { Elaboración de Organizadores } \\
\text { Gráficos (SINTESIS) }\end{array}$ & 9,0 & 9,0 & 7,0 & 8,0 & 9,0 & 10,0 & 9,0 & 8,0 & 10,0 & 9,5 & 8,0 & 9,0 & 9,5 & 9,0 & 8,5 & 9,0 & 9,0 & 9,5 \\
\hline $\begin{array}{l}\text { Proyecto (SOLUCION DE } \\
\text { PROBLEMAS, CAPACIDAD } \\
\text { DE TRABAJOEN EQUIPO, } \\
\text { INVESTIGACION) }\end{array}$ & 10,0 & 10,0 & 10,0 & 10,0 & 10,0 & 10,0 & 10,0 & 10,0 & 9,5 & 9,5 & 9,5 & 9,5 & 10,0 & 10,0 & 10,0 & 10,0 & 8,0 & 8,0 \\
\hline $\begin{array}{c}\text { Prueba Final (APLICACION Y } \\
\text { RAZONAMIENTO) }\end{array}$ & 9,0 & 9,0 & 9,5 & 10,0 & 8,0 & 8,0 & 8,0 & 10,0 & 9,0 & 8,0 & 9,0 & 7,0 & 9,0 & 9,5 & 9,0 & 10,0 & 8,0 & 9,0 \\
\hline SUMA & 47,0 & 48,0 & 44,0 & 45,0 & 43,0 & 46,5 & 46,0 & 46,0 & 46,0 & 44,0 & 44,5 & 44,5 & 47,5 & 45,5 & 45,5 & 47,0 & 45,0 & 45,0 \\
\hline PROMEDIO & 9,4 & 9,6 & 8,8 & 9 & 8,6 & 9,3 & 9,2 & 9,2 & 9,2 & 8,8 & 8,9 & \begin{tabular}{|l|l}
8,9 \\
\end{tabular} & 9,5 & 9,1 & 9,1 & 9,4 & 9 & 9 \\
\hline
\end{tabular}

Fuente: elaboración propia.

Tabla 2. Promedio Paralelo $A=9,1$.

\begin{tabular}{|c|c|c|c|c|c|c|c|c|c|c|c|c|c|c|c|c|c|c|c|c|}
\hline № de Estudiante & 19 & 20 & 21 & 22 & 23 & 24 & 25 & 26 & 27 & 28 & 29 & 30 & 31 & 32 & 33 & 34 & 35 & 36 & SUMA & PROMEDIO \\
\hline \multicolumn{21}{|l|}{ INSTRUMENTO/INDICADOR } \\
\hline $\begin{array}{c}\text { Laboratorios (ANALISIS Y } \\
\text { APLICACION) }\end{array}$ & 10,0 & 10,0 & 8,0 & 9,0 & 9,0 & 9,0 & 9,5 & 9,0 & 9,8 & 8,0 & 8,5 & 9,0 & 9,5 & 9,0 & 8,0 & 9,5 & 9,0 & 10,0 & 322,8 & 8,97 \\
\hline Foros (RAZONAMIENTO) & 9,5 & 9,0 & 10,0 & 8,0 & 7,0 & 10,0 & 8,5 & 10,0 & 7,0 & 9,0 & 8,0 & 10,0 & 9,0 & 8,0 & 9,0 & 9,0 & 9,0 & 9,5 & 327,5 & 9,10 \\
\hline $\begin{array}{l}\text { Elaboración de Organizadores } \\
\text { Gráficos (SINTESIS) }\end{array}$ & 10,0 & 9,0 & 9,0 & 9,0 & 8,0 & 8,0 & 9,0 & 9,0 & 9,0 & 8,0 & 9,0 & 9,0 & 9,5 & 9,0 & 9,5 & 9,0 & 8,0 & 10,0 & 321,0 & 8,92 \\
\hline $\begin{array}{l}\text { Proyecto (SOLUCION DE } \\
\text { PROBLEMAS, CAPACIDAD } \\
\text { DE TRABAJO EN EQUIPO, } \\
\text { INVESTIGACION) }\end{array}$ & 8,0 & 8,0 & 9,0 & 9,0 & 9,0 & 9,0 & 10,0 & 10,0 & 10,0 & 10,0 & 9,5 & 9,5 & 9,5 & 9,5 & 10,0 & 10,0 & 10,0 & 10,0 & 344,0 & 9,56 \\
\hline $\begin{array}{l}\text { Prueba Final (APLICACION Y } \\
\text { RAZONAMIENTO) }\end{array}$ & 10,0 & 9,0 & 9,0 & 8,0 & 9,0 & 8,0 & 10,0 & 9,0 & 8,0 & 10,0 & 9,0 & 10,0 & 9,0 & 10,0 & 9,0 & 9,0 & 7,0 & 10,0 & 322,0 & 8,94 \\
\hline SUMA & 47,5 & 45,0 & 45,0 & 43,0 & 42,0 & 44,0 & 47,0 & 47,0 & 43,8 & 45,0 & 44,0 & 47,5 & 46,5 & 45,5 & 45,5 & 46,5 & 43,0 & 49,5 & 1637,3 & 45,48 \\
\hline PROMEDIO & 9,5 & 9 & 9 & 8,6 & 8,4 & 8,8 & 9,4 & 9,4 & 8,76 & 9 & 8,8 & 9,5 & 9,3 & 9,1 & 9,1 & 9,3 & 8,6 & 9,9 & 327,5 & 9,10 \\
\hline
\end{tabular}

Fuente: elaboración propia. 


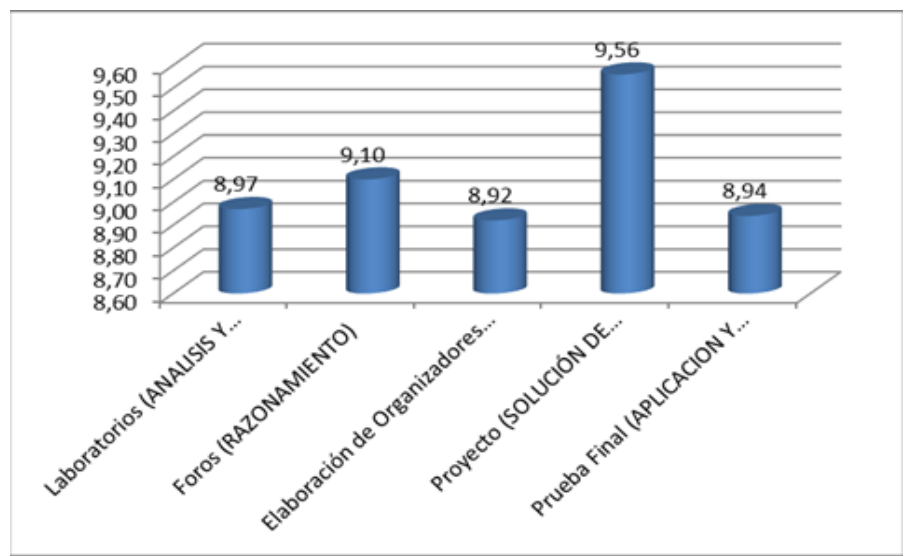

Gráfico 1. Instrumentos indicadores vs Promedio de rendimiento académico paralelo A. Fuente: elaboración propia.

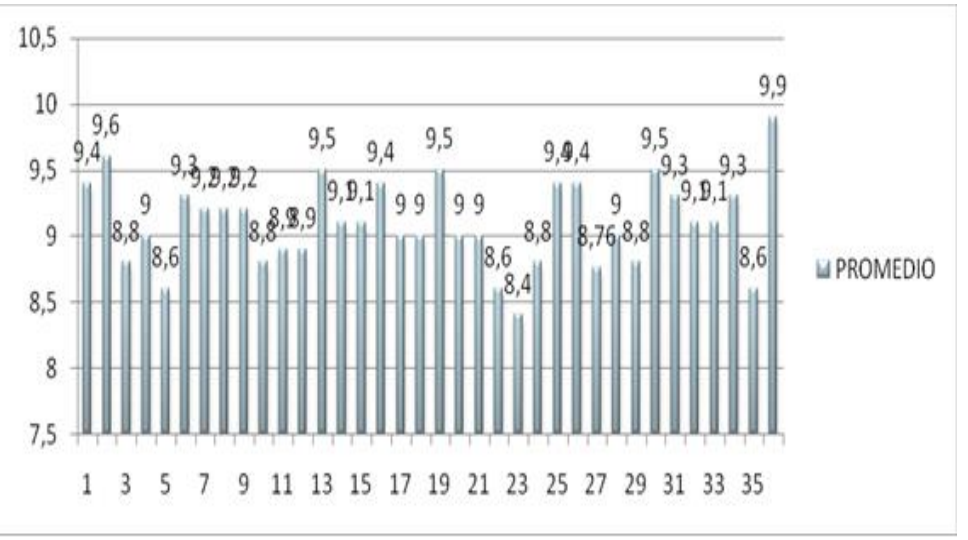

Gráfico 2. Estudiantes paralelo A vs Rendimiento Académico.

Fuente: elaboración propia.

\subsubsection{Datos obtenidos con el Paralelo B.}

Los datos que se muestran a continuación se obtuvieron aplicando los instrumentos de evaluación pertinente a los 34 alumnos del paralelo "B".

Tabla 3. Datos obtenidos con el paralelo B al aplicar los instrumentos de evaluación.

\begin{tabular}{|c|c|c|c|c|c|c|c|c|c|c|c|c|c|c|c|c|c|c|c|c|}
\hline № de Estudiante & 1 & 2 & 3 & 4 & 5 & 6 & 7 & 8 & 9 & 10 & 11 & 12 & 13 & 14 & 15 & 16 & 17 & 18 & 19 & 20 \\
\hline \multicolumn{21}{|l|}{ INSTRUMENTO/INDICADOR } \\
\hline $\begin{array}{c}\text { Laboratorios (ANALISIS Y } \\
\text { APLICACION) }\end{array}$ & 9,0 & 9,0 & 7,0 & 9,5 & 8,0 & 9,0 & 8,0 & 8,0 & 9,0 & 8,0 & 9,0 & 10,0 & 9,0 & 8,0 & 9,0 & 9,0 & 10,0 & 9,0 & 10,0 & 10,0 \\
\hline Foros (RAZONAMIENTO) & 7,0 & 6,0 & 8,0 & 8,0 & 7,0 & 8,0 & 7,0 & 8,0 & 8,0 & 7,0 & 6,0 & 6,0 & 7,0 & 6,0 & 7,0 & 8,0 & 9,0 & 7,0 & 6,0 & 7,0 \\
\hline $\begin{array}{c}\text { Elaboración de Organizadores } \\
\text { Gráficos (SINTESIS) }\end{array}$ & 7,0 & 9,0 & 5,0 & 8,0 & 8,5 & 8,0 & 8,0 & 8,0 & 7,5 & 7,0 & 7,5 & 7,0 & 8,0 & 8,0 & 8,5 & 8,5 & 8,0 & 8,0 & 8,5 & 8,0 \\
\hline $\begin{array}{l}\text { Proyecto (SOLUCION DE } \\
\text { PROBLEMAS, CAPACIDAD } \\
\text { DE TRABAJO EN EQUIPO, } \\
\text { INVESTIGACION) }\end{array}$ & 8,0 & 8,0 & 8,0 & 8,0 & 9,0 & 9,0 & 9,0 & 9,0 & 8,0 & 8,0 & 8,0 & 8,0 & 8,5 & 8,5 & 8,5 & 8,5 & 9,0 & 9,0 & 9,0 & 9,0 \\
\hline $\begin{array}{c}\text { Prueba Final (APLICACION Y } \\
\text { RAZONAMINTO) }\end{array}$ & 7,0 & 8,0 & 9,0 & 8,0 & 7,0 & 8,0 & 7,5 & 7,5 & 9,0 & 8,0 & 7,0 & 6,0 & 8,0 & 7,0 & 5,0 & 4,0 & 6,0 & 8,0 & 6,0 & 5,0 \\
\hline SUMA & 38,0 & 40,0 & 37,0 & 41,5 & 39,5 & 42,0 & 39,5 & 40,5 & 41,5 & 38,0 & 37,5 & 37,0 & 40,5 & 37,5 & 38,0 & 38,0 & 42,0 & 41,0 & 39,5 & 39,0 \\
\hline PROMEDIO & 7,6 & 8,0 & 7,4 & 8,3 & 7,9 & 8,4 & 7,9 & 8,1 & 8,3 & 7,6 & 7,5 & 7,4 & 8,1 & 7,5 & 7,6 & 7,6 & 8,4 & 8,2 & 7,9 & 7,8 \\
\hline
\end{tabular}

Fuente: elaboración propia. 


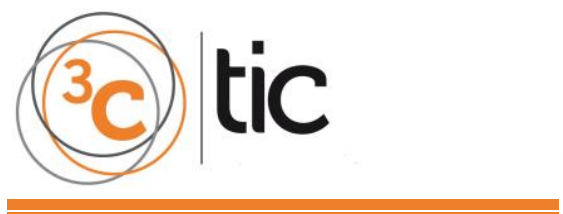

Tabla 4. Promedio Paralelo $B=7,42$.

\begin{tabular}{|c|c|c|c|c|c|c|c|c|c|c|c|c|c|c|c|c|}
\hline № de Estudiante & 21 & 22 & 23 & 24 & 25 & 26 & 27 & 28 & 29 & 30 & 31 & 32 & 33 & 34 & SUMA & PROMEDIO \\
\hline \multicolumn{17}{|l|}{ INSTRUMENTO/INDICADOR } \\
\hline $\begin{array}{c}\text { Laboratorios (ANALISIS Y } \\
\text { APLICACION) }\end{array}$ & 8,0 & 9,0 & 9,0 & 9,0 & 9,5 & 9,0 & 9,8 & 8,0 & 8,5 & 9,0 & 9,5 & 9,0 & 8,0 & 9,5 & 302,3 & 8,89117647 \\
\hline Foros (RAZONAMIENTO) & 8,0 & 7,0 & 6,0 & 8,0 & 7,0 & 7,0 & 6,0 & 8,0 & 6,0 & 7,0 & 7,5 & 7,0 & 6,0 & 6,5 & 240,0 & 7,05882353 \\
\hline $\begin{array}{l}\text { Elaboración de Organizadores } \\
\text { Gráficos (SINTESIS) }\end{array}$ & 8,0 & 9,0 & 8,0 & 8,0 & 7,5 & 8,0 & 8,0 & 8,5 & 8,0 & 7,0 & 8,0 & 7,0 & 9,0 & 8,0 & 268,0 & 7,88235294 \\
\hline $\begin{array}{c}\text { Proyecto (SOLUCION DE } \\
\text { PROBLEMAS, CAPACIDAD } \\
\text { DE TRABAJO EN EQUIPO, } \\
\text { INVESTIGACION) }\end{array}$ & 9,0 & 9,0 & 9,0 & 9,5 & 8,5 & 8,5 & 8,5 & 8,5 & 9,0 & 9,0 & 9,0 & 9,5 & 8,0 & 9,0 & 294,0 & 8,64705882 \\
\hline $\begin{array}{l}\text { Prueba Final (APLICACION Y } \\
\text { RAZONAMIENTO) }\end{array}$ & 9,0 & 7,0 & 6,0 & 8,0 & 5,0 & 7,0 & 8,0 & 4,0 & 5,0 & 7,0 & 8,0 & 7,0 & 6,0 & 5,5 & 233,5 & 6,86764706 \\
\hline SUMA & 42,0 & 41,0 & 38,0 & 42,5 & 37,5 & 39,5 & 40,3 & 37,0 & 36,5 & 39,0 & 42,0 & 39,5 & 37,0 & 38,5 & 1337,8 & 39,35 \\
\hline PROMEDIO & 8,4 & 8,2 & 7,6 & 8,5 & 7,5 & 7,9 & 8,1 & 7,4 & 7,3 & 7,8 & 8,4 & 7,9 & 7,4 & 7,7 & 267,6 & 7,87 \\
\hline
\end{tabular}

Fuente: elaboración propia.

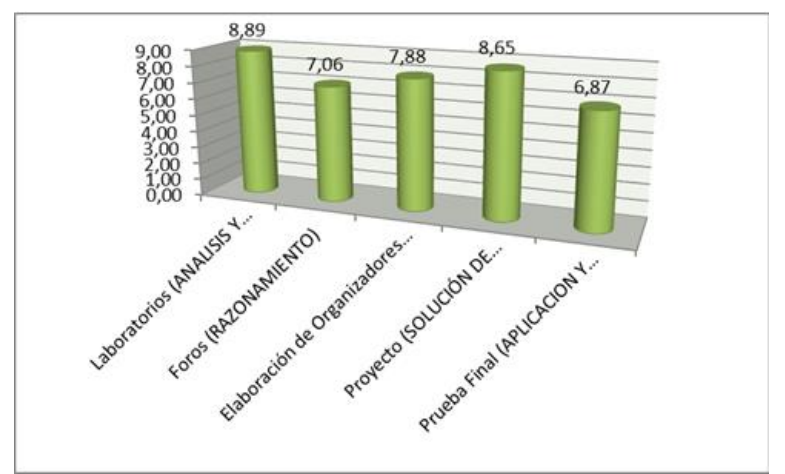

Gráfico 3. Instrumentos indicadores vs Promedio de rendimiento académico paralelo B. Fuente: elaboración propia.

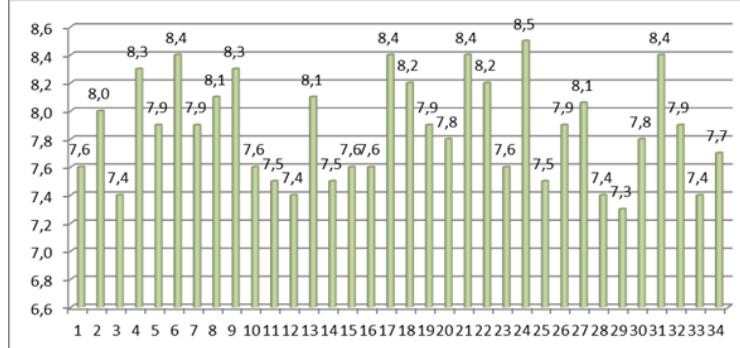

Gráfico 4. Estudiantes paralelo B vs Rendimiento académico.

Fuente: elaboración propia.

\subsubsection{Presentación de los resultados en cuadros y/o gráficos.}

Una vez realizado el análisis de los datos podemos establecer los siguientes resultados.

\section{Población}

La población está conformada por todos los estudiantes de la Escuela de Sistemas y Tecnologías de la Universidad Técnica de Babahoyo. Muestra 70 alumnos del Quinto Semestre. 
Paralelo $A=36$ estudiantes

Paralelo $B=34$ estudiantes

Total 70 estudiantes

$$
\begin{array}{ll}
\text { Muestra: } & \mathrm{NA}=36 \text { estudiantes } \\
& \mathrm{NB}=34 \text { estudiantes }
\end{array}
$$

Tabla 5. Tabla comparativa Paralelo A vs Paralelo B.

\begin{tabular}{ccc}
\hline Caracteristica & Paralelo B & Paralelo A \\
\hline $\begin{array}{c}\text { Media de las } \\
\text { muestras }\end{array}$ & 7,87 & 9,10 \\
$\begin{array}{c}\text { Desviación } \\
\text { Estándar } \\
\text { Tamaño de la } \\
\text { Muestra }\end{array}$ & 0,32 & 0,36 \\
\hline
\end{tabular}

Fuente: elaboración propia.

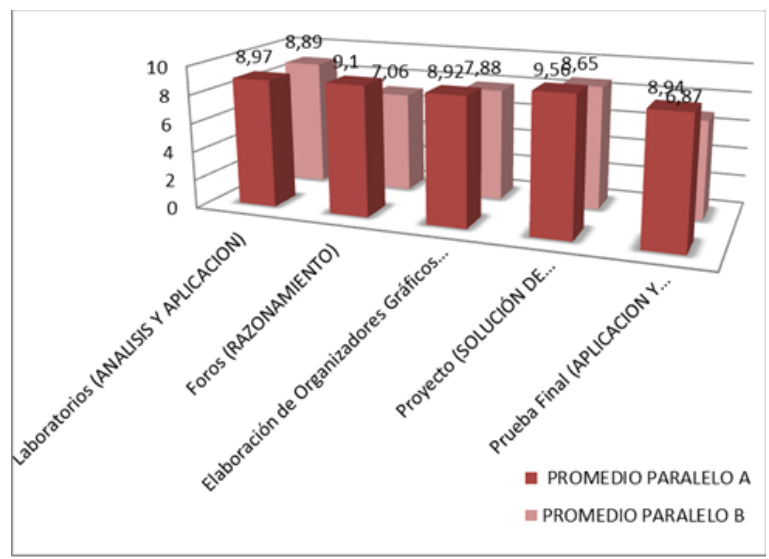

Gráfico 5. Cuadro comparativo del promedio de cada instrumento Paralelo A vs Paralelo B.

Fuente: elaboración propia.

Se puede notar que en el paralelo A supera al paralelo B, en los resultados comparativos de promedios de rendimiento académico, por lo tanto en cuanto a razonamiento, análisis y aplicación fueron mucho mejores los resultados del grupo cuasi experimental. 


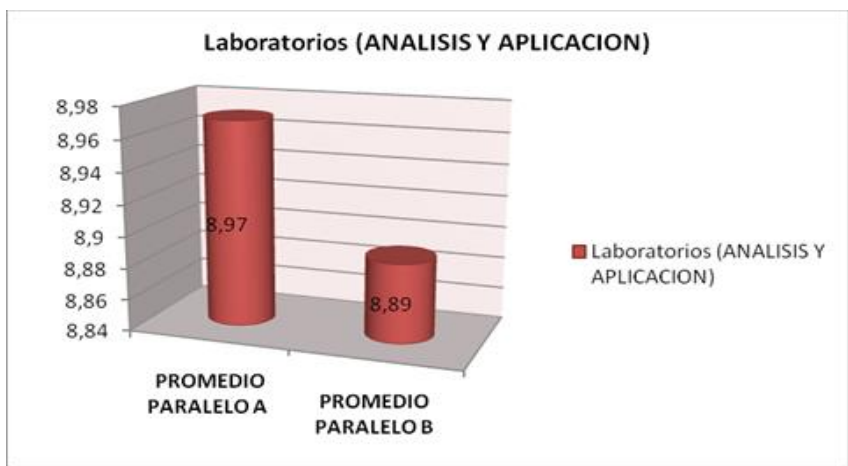

Gráfico 6. Cuadro comparativo Instrumento 1 Paralelo A vs Paralelo B.

Fuente: elaboración propia.

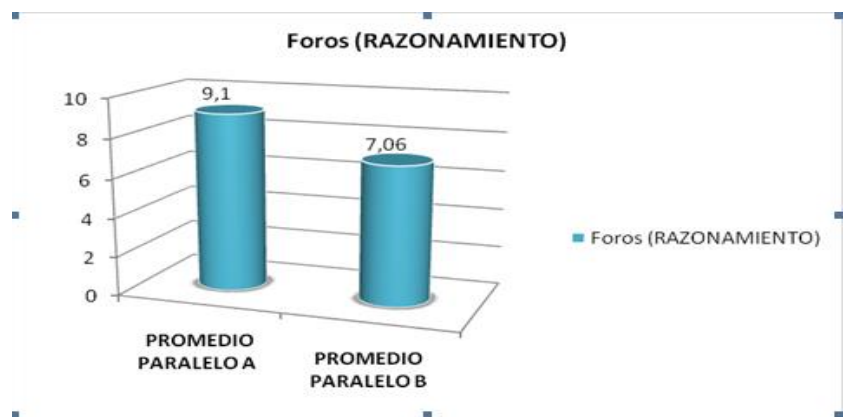

Gráfico 7. Cuadro comparativo Instrumento 2 Paralelo A vs Paralelo B.

Fuente: elaboración propia.

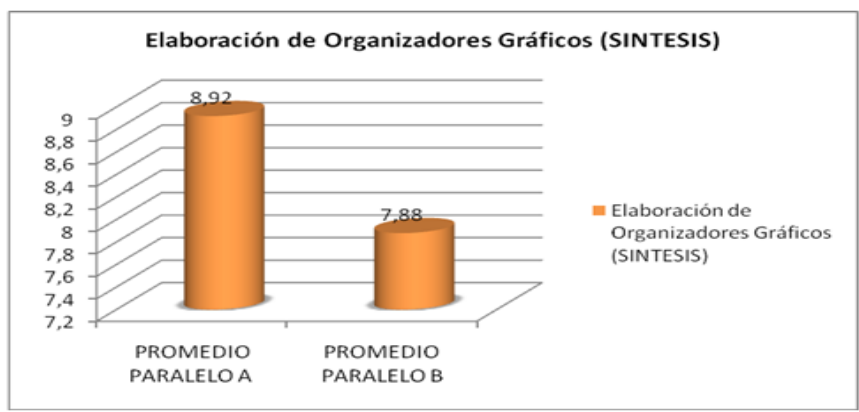

Gráfico 8. Cuadro comparativo Instrumento 3 Paralelo A vs Paralelo B. Fuente: elaboración propia.

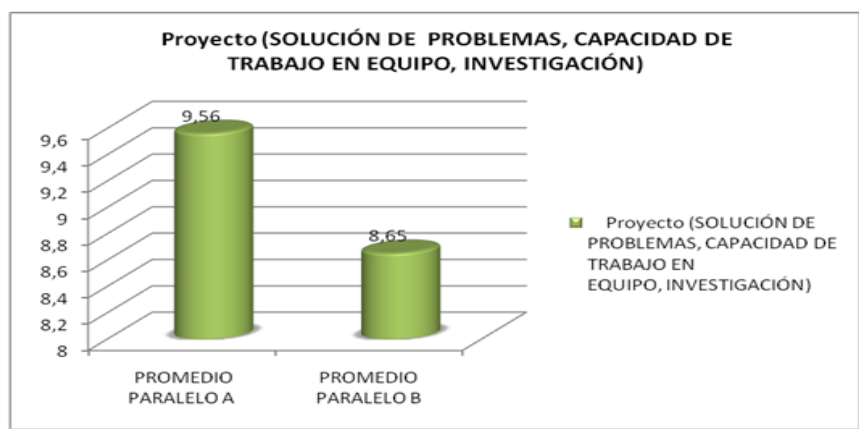

Gráfico 9. Cuadro comparativo Instrumento 4 Paralelo A vs Paralelo B.

Fuente: elaboración propia. 


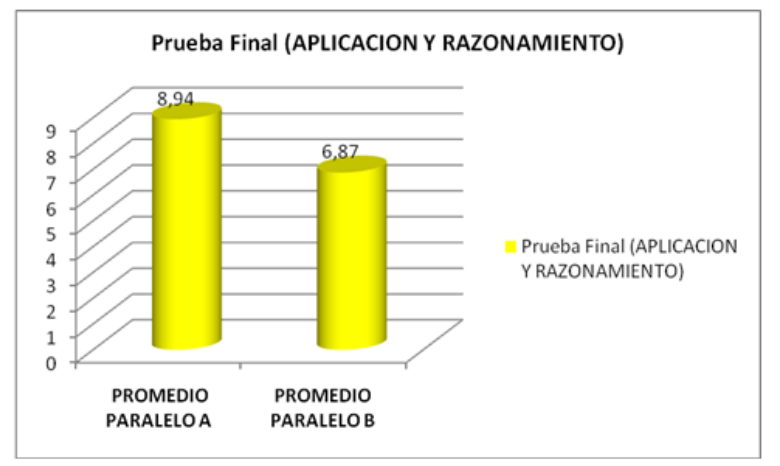

Gráfico 10. Cuadro comparativo Instrumento 5 Paralelo A vs Paralelo B.

Fuente: elaboración propia.

\subsection{PRUEBA DE HIPÓTESIS}

Antes de poner en ejecución la propuesta, se determinó que los dos grupos estén en condiciones similares. Para ello se tomaron los resultados de la evaluación final de semestre, es decir los promedios finales de cada alumno en la Asignatura de Redes de Computadores.

\subsubsection{Planteamiento de la Hipótesis $\mathrm{H} 1$}

H1: La utilización de mundos virtuales de aprendizaje permitirá mejorar el nivel de rendimiento académico en la Asignatura de Redes de computadores.

H0: La utilización de mundos virtuales de aprendizaje no permitirá mejorar el nivel de rendimiento académico en la Asignatura de Redes de computadores.

HO: $\mu b \leq \mu \mathrm{a}$

H1: $\mu \mathrm{a}>\mu \mathrm{b}$

Tabla 6. Valores críticos de z para pruebas de una o dos colas.

\begin{tabular}{|c|c|r|r|r|r|}
\hline Nivel de significación & 0.10 & \multicolumn{1}{c|}{0.05} & 0.010 & 0.005 & 0.002 \\
\hline Valores críticos de z para & -1.28 & -1.645 & -2.33 & -2.58 & -2.88 \\
pruebas a una cola & 01.28 & 01.645 & $\circ 2.33$ & $\circ 2.58$ & $\circ 2.88$ \\
\hline Valores críticos de z para & -1.645 & -1.96 & -2.58 & -2.81 & -3.08 \\
pruebas a dos colas & y 1.645 & y 1.96 & y 2.58 & y 2.81 & y 3.08 \\
\hline
\end{tabular}

Fuente: elaboración propia.

\subsubsection{Nivel de significación}

$\alpha=0.05$

Rechácese H0 si $-1,96 \geq z \geq 1,96$ 


\subsubsection{Determinación de la región de rechazo}

- H0 se rechaza si $-1,96 \leq z \geq 1,96$

\subsubsection{Cálculos}

$$
\begin{aligned}
& z=\frac{\overline{X_{a}}-\overline{X_{b}}}{\sqrt{\frac{\delta a^{2}}{n a}+\frac{\delta b^{2}}{n b}}} \\
& z=\frac{9.10-7,87}{\sqrt{\frac{(0,32)^{2}}{36}+\frac{(0,36)^{2}}{34}}} \quad z=\frac{1,23}{\sqrt{\frac{0,10}{36}+\frac{0.13}{34}}} \\
& z=\frac{1,23}{\sqrt{0,0028+0,0038}} \\
& z=\frac{1,23}{\sqrt{0,01}} \\
& z=\frac{1,23}{0,08}
\end{aligned}
$$

Como el 15,08 > 1,96 entonces se rechaza la Hipótesis Nula y se acepta la hipótesis de investigación: "El nivel de rendimiento académico de los estudiantes que utilizan Plataformas 


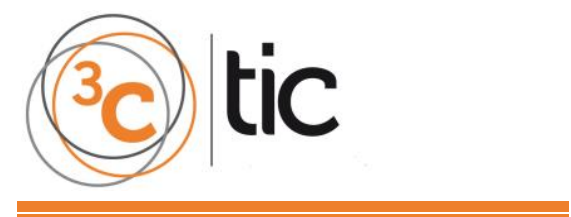

Virtuales en el proceso de aprendizaje de la asignatura Redes de Computadores supera el nivel de rendimiento Académico de los estudiantes que no las utilizan".

\section{CONCLUSIONES}

- La implementación del mundo virtual de aprendizaje en la asignatura redes de computadores en la UTB, permitió mejorar la asimilación del contenido de dicha asignatura por parte de los alumnos.

- La utilización de herramientas síncronas y asíncronas permiten obtener un mejor aprovechamiento de los recursos compartidos por los participantes del mundo virtual de aprendizaje.

- La utilización de mundos virtuales de aprendizaje proporcionan una poderosa herramienta de transmisión de conocimientos, posicionando a la UTB en un sitial privilegiado al poseer una tecnología de vanguardia.

- Se desarrolló una guía de configuración e implementación del mundo virtual de aprendizaje de la UTB que permitirá la implementación de cursos similares en nuestra alma mater.

\section{RECOMENDACIONES}

- La implementación de mundos virtuales demanda de un alto consumo de recursos hardware, así como también de ancho de banda de red para que se ofrezca un servicio óptimo y sin retardos considerables.

- El servicio de alojamiento de sitios virtuales comerciales tiene un costo considerable, la compra de la región tiene un precio de 1100 dólares y las mensualidades por mantenimiento oscila en un promedio de 250 dólares.

- Se debe dar el mantenimiento adecuado al sitio para no dejar que la información se haga obsoleta y eliminar los cursos que ya han sido impartidos.

- Es primordial realizar la difusión del entorno para que los docentes de la UTB se familiaricen con la tecnología y adecuen sus cursos a la plataforma de aprendizaje virtual.

- Se debe tomar en consideración la creación del campus virtual propio de la Universidad Técnica de Babahoyo, ya muchas instituciones a nivel internacional lo poseen y están sacando el máximo provecho de aquello.

- Se debería profundizar en la utilización de la realidad virtual inmersiva muy utilizada en juegos actualmente con la utilización de gafas 3D y que Second Life ya soporta permitiendo hacer una experiencia más real. 


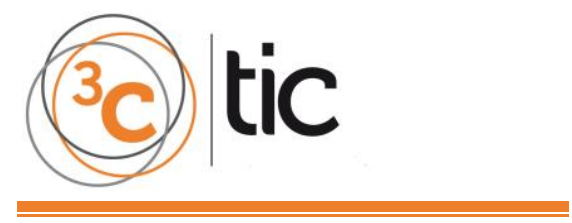

\section{REFERENCIAS BIBLIOGRÁFICAS}

Barberà E. (2006) Los fundamentos teóricos de la tutoría presencial y en línea: una perspectiva socio-constructivista, en Jerónimo y Aguilar (2006) Educación en Red y Tutoría en Línea, UNAM FES Zaragoza, México.

Bonk, C. J., y Cunningham, D. J. (1998). "Searching for learner-centered, constructivist, and sociocultural components of collaborative educational learning tools". In C. J. Bonk \& K. S. Kim (Eds.), Electronic collaborators: learner-centered technologies for literacy, apprenticeship, and discourse pp. 25-50. New Jersey: Erlbaum.

Castronova, E. (2006). Synthetic worlds: the business and culture of online games, The University of Chicago Press, Chicago and London.

Crichton S., Kinash S., (2003) Virtual Ethnography: Interactive Interviewing Online as Method. Canadian Journal of Learning and Technology 29(2)

Dickey M. (2003) Teaching in 3D: Pedagogical Affordances and Constraints of 3D Virtual Worlds for Synchronous Distance Learning. Distance Education, 24 (1)

Hine C. (2000) Virtual Ethnography. Sage, Londres.

Hollander y Thomas (2009) Commentary: Virtual Planning: Second Life and the Online Studio. Journal of Planning Education and Research. 29; 108

Garrison R. (2000) Theoretical Challenges for Distance Education in the 21st Century: A Shift from Structural to Transactional Issues. International Review of Research in Open and Distance Learning. 1(1)

Garrison y Anderson (2003) E-learning in the 21st. Century. Routledge Falmer. New York.

Gros S.B (2004) La construcción del conocimiento en la Red, límites y posibilidades. Teoría de la Educación: Educación y Cultura en la Sociedad de la Información. (5)

Recuperado

de:

<http://www3.usal.es/ teoriaeducacion/rev numero 05/n5 articulos.htm/>.

Consultada en mayo de 2009

Geertz, C. (1995) Descripción densa: hacia una teoría interpretativa de la cultura. En Geertz, La interpretación de las culturas Gedisa (pp.19-40). España.

Giddens Anthony (1993) Consecuencias de la modernidad. Madrid. Alianza.

Giddens, Anthony (1997) “Los contornos de la Modernidad" en: Modernidad e identidad del yo. El yo y la sociedad en la época contemporánea. Península, Barcelona.

Giddens, Anthony (2000) Un mundo desbocado: los efectos de la globalización en nuestras vidas. Santillana. España. 


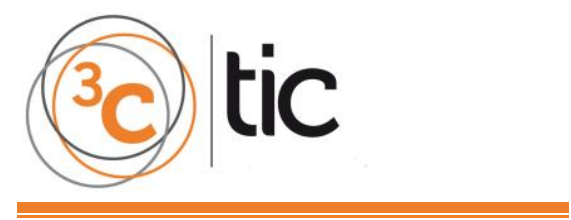

Hara N., Bonk C., Angeli Ch. (2000) Content Analysis of Online Discussion en an applied Educational Psychologogy, CRLT Technical Report, Instructional Science, No. 2-98, USA

Hine C. (2000) Virtual Ethnography. Sage, Londres; (2004) para la versión en español, Etnografía Virtual, UOC. Barcelona.

Jones S. (2002) Virtual Culture, Identity and Communication in Cybersociety, Sage. Londres.

Klastrup L. (2003) A Poetics of Virtual Worlds, Proceedings of Digital Arts and Culture, Melbourne, Australia 2003.: 100-109.

Lombardi, J., y McCahill, M. P. (2004) Enabling social dimensions of learning through a persistent, unified, massively multi-user, and self-organizing virtual environment. Creating, Connecting and Collaborating through Computing, 2, 166-172.

Mann Ch. y Stewardt F. (2002) Internet Communication and Qualitative Research, Sage. Inglaterra.

Mc Louglin, C., Winnips, JC., Oliver R. (2000) Supporting constructivist learning through learnes support on-line. EDUMEDIA

Molka-Danielsen, J., Carter, B., Richardson, D., and Jæger, B. (2009).Teaching and Learning Affectively within a Virtual Campus, (eds.) Birch, D. and Al-Hakim, L. en Special Issue: Towards Virtual Universities of the International Journal of Networking and Virtual Organisations. Inderscience Enterprises, Ltd

Murdock, G. (1993) "Communications and the constitution of modernity", Media, Culture and Society, vol. 15

Otero V.K (2003) Cognitive processes and the learning of physics part II: mediated action Proceedings of the International School of Physics "Enrico Fermi", editedado por M. Vicentini y E.F. Redish (IOS Press, Amsterdam), Varenna, Italia

Riviere, A. (1988) La psicología de Vigotsky, Visor. Madrid.

Rourke I, Anderson T, Garrison To, Archer W. (2001) Social Assessing Presence In Asynchronous Text-based Computer Conferencing Journal of Distance Education/Revue of l'enseignement à distance.

Schrage, M. (1990). Shared minds: The technologies of collaboration. New York: Random House. En Hara N. et al (2000) CRLT Technical Report No. 2-98 Content Analysis of OnlineDiscussion in an Applied Educational Psychology.

Stephenson, Neal(1992) Snow Crash. Gigamesh. Barcelona

Turkle S. (1995) La vida en pantalla: La identidad en la era de Internet, Paidós. España.

Twining P. (2009) Exploring the educational potential of virtual worlds-Some reflections from the SPP. British Journal of Educational Technology. 40 (3) 
Waskul, D.D. (2003) Self-Games and Body-play: personhood in on-line chat and cybersexes, Peter Lang, NY.

Wegerif, R. "The social dimension of Asynchronous Learning Networks", 1998. Recuperado de: <www.aln.org/alnweb/journal/vol2 issue1/wegerif.htm/>. Consultado en enero, 2003.

Zapata R. M. (2001) "Formación abierta y a distancia a través de redes digitales: Modelos de redes de aprendizaje". <http://www.um.es/ead/red/1/red1.htm/>. Consultado en julio, 2001.

Zubero I. (1998). "Participación y democracia ante las nuevas tecnologías. Retos políticos de la sociedad de la información".

[en linea]. <http://www.adi.uam.es / jparedes/ lecturas/zubero.html/>. consultado en mayo de 2009. 